\title{
Orobanche rameuse (Orobanche ramosa L.) du colza : un risque émergent sous surveillance
}

Stéphanie GIBOT-LECLERC ${ }^{1}$

Xavier PINOCHET ${ }^{2}$

Georges SALLÉ ${ }^{1}$

${ }^{1}$ Laboratoire de parasitologie végétale, case courrier 155, Université Pierre et Marie Curie, 4 Place Jussieu, 75252 Paris cedex 05, France ${ }^{2}$ CETIOM, Direction scientifique, $B P n^{\circ} 4$, 78850 Thiverval-Grignon France <pinochet@cetiom.fr>

\begin{abstract}
Broomrapes are parasitic plants without chlorophyll, nutritionally depending from their host plant. Orobanche ramosa L. is a parasite of numerous plants including winter oilseed rape. Its presence has been reported for a long time in the South of France. Nevertheless, its presence became a major problem in the Central West region Poitou-Charentes since the middle of the nineties. The phenomenon is under observation, and research work has been undertaken to evaluate its extension risks and to propose efficient solutions. The main extension factors are its ability to produce seeds, its wide ability to germinate in different environmental conditions, its large number of potential host plants, including common weeds present in cropped fields. Short term recommendations intend to favour the host in the nutritional competition and to limit the soil seed bank. For a longer term, several possibilities could be carried out: optimization of false hosts/trap-crops introduction in the rotation which could decrease the soil seed bank; and breeding for herbicide resistant plants through mutagenesis or genetic transformation.
\end{abstract}

Key words: broomrape, parasite, oilseed rape, extension risk, host plant, control methods

Les orobanches sont des angiospermes dépourvues de chlorophylle qui parasitent de nombreuses plantes cultivées du Moyen-Orient et du Bassin méditerranéen pour leur alimentation en eau, en éléments minéraux, en carbone et en azote. Parmi les nombreuses espèces du genre Orobanche, on peut citer O. cumana Wallr., parasite du tournesol en Espagne et en Turquie, ainsi qu'O. crenata Forsk., parasite de légumineuses à graines comme la fève ou le pois chiche. 0 . ramosa possède de nombreux hôtes dont le colza et des plantes d'intérêt économique comme la tomate, la pomme de terre, le tabac ou le chanvre. Elle est répertoriée de longue date dans le sud de la France, y compris dans les cultures de colza [1] et elle est devenue gênante sur le tabac dès les années 1950 [2]. Sa présence devient préoccupante sur certaines zones de la région Poitou-Charentes depuis le milieu des années 90, en particulier dans le secteur de St-Jean-d'Angély (Charente-Maritime). L'émergence puis la confirmation de ces difficultés ont incité le CETIOM à mettre en place une surveillance régulière et à engager, avec des équipes de spécialistes, des travaux ayant pour principal objectif de cerner les risques d'extension du parasite et d'ébaucher des solutions de contrôle de son impact. L'objet de cette synthèse est de présenter les principaux résultats obtenus ces dernières années dans le cadre d'une collaboration entre le CETIOM et l'équipe du laboratoire de parasitologie végétale de l'université Pierre et Marie Curie.

\section{Les observations de terrain}

Outre les remontées d'information de différents acteurs de terrain, le CETIOM a mis en place depuis 1999 des enquêtes kilométriques ayant pour objet, sur un parcours prédéterminé dans une région, de visiter les parcelles de colza rencontrées à écart de distance fixé afin de constater la présence ou l'absence du parasite. La figure 1 montre l'évolution du phénomène dans le secteur géographique de St-Jean-d'Angély (17, Charente-Maritime), où il est le plus préoccupant.

La présence de l'orobanche a également été observée dans d'autres zones de la région PoitouCharentes. L'étude de l'extension à l'échelle régionale par des enquêtes kilométriques a permis de mettre en évidence un secteur également très contaminé : le triangle défini par les villes de Fontenay-le-Comte (85, Vendée), Niort (79, Deux-Sèvres), et St-Maixent-l'école (79, DeuxSèvres). On trouve également des parcelles sévèrement parasitées dans le nord du département des Deux-Sèvres, entre Partenay et Thouard. Par ailleurs, une enquête récemment conduite dans le cadre d'un groupe de travail de l'AFPP (Association française de protection des plantes) sur les plantes envahissantes indique que l'orobanche est signalée dans 74 réponses (13\%), indiquant sa présence dans de nombreuses régions telles que Midi-Pyrénées, Aquitaine, Poitou-Charentes, mais aussi Centre, Bourgogne, Auvergne, Pays-de-Loire, Champagne, Franche-Comté et Alsace [3]. Ces données devraient bientôt être confirmées par le dispositif de suivi de l'évolution des flores des zones cultivées que met en place le ministère de l'Agriculture (DGAL-SDPV). Ce dispositif prévoit de suivre, par an, 1000 parcelles au sein desquelles la présence de quelques espèces réputées envahissantes, dont l'orobanche rameuse, sera systématiquement recherchée.
FONDAMENTAL
Article reçu le 21 juillet 2005

Accepté le 21 février 2006 


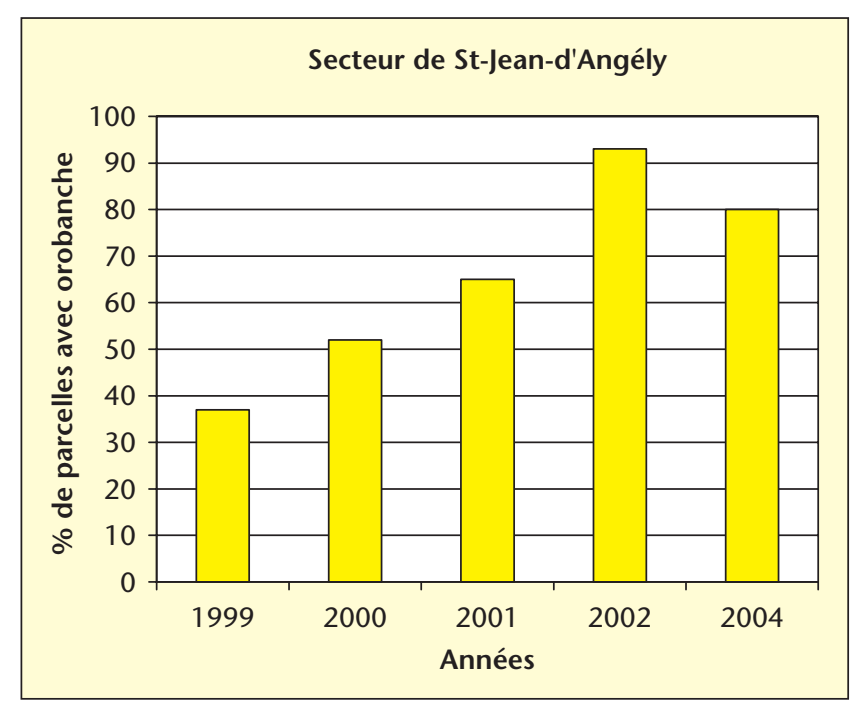

Figure 1. Résultats des enquêtes kilométriques conduites par le CETIOM dans le secteur de St-Jean-d'Angély.

\section{Les principaux facteurs d'extension}

Les travaux réalisés par Gibot-Leclerc [4] permettent de distinguer quatre facteurs de risques principaux de l'extension du parasitisme colza/orobanche rameuse.

\section{La production de graines}

Le premier facteur est le niveau très élevé de production de graines de l'espèce. Chaque hampe florale d'orobanche est capable de disséminer de $10^{5}$ à $10^{6}$ graines [4]. Dans un champ cultivé, chaque pied de colza peut permettre le développement de 20 à 30 hampes florales du parasite. De plus, le poids de mille graines de l'orobanche est faible (3 à 10 milligrammes) et la dissémination des graines, ainsi fortement facilitée, est assimilable à celles du pollen ou de spores de champignons pathogènes aériens.

\section{Germination}

Contrairement à l'étude de Saghir [5], Gibot-Leclerc [4] a montré qu'il n'existe pas de dormance primaire chez les graines d'orobanche rameuse récoltées sur différentes années en région Poitou-Charentes et soumises à un préconditionnement de 14 jours à $20^{\circ} \mathrm{C}$ (tableaux 1 et 2). Par ailleurs, ces semences gardent une très bonne aptitude à germer durant au moins 3 années (tableau 3). Une dormance secondaire peut s'installer si le préconditionnement dure trop longtemps, sans qu'il y ait initiation de la germination par les exsudats racinaires de la plante hôte ou par un inducteur chimique tel les analogues du strigol (GR 7, GR 24, GR 41). Ainsi, une dormance secondaire a été obtenue après 100 jours de préconditionnement aux températures suboptimales de préconditionnement $\left(5\right.$ et $30^{\circ} \mathrm{C}$ ) mais aucune après un préconditionnement de 100 jours à $20^{\circ} \mathrm{C}$ (température optimale de préconditionnement). Les facteurs du milieu agissent sur le préconditionnement, même s'ils sont peu déterminants. Le préconditionnement des graines est donc effectif pour des températures comprises entre 5 et $30^{\circ} \mathrm{C}$. À $20^{\circ} \mathrm{C}$, le préconditionnement ne peut avoir lieu en anoxie complète mais il est effectif pour des tensions en oxygène comprises entre 1 et $21 \%$ et dans une gamme de potentiels hydriques allant de 0 à $-2 \mathrm{MPa}$. Dès l'instant où les graines sont préconditionnées, il peut y avoir induction de la germination par les exsudats racinaires de la plante hôte. Les études menées sur différents hôtes de l'orobanche et de striga (Striga sp. Scrophulariacée africaine,
Tableau 1. Fréquences de germination observées immédiatement après la récolte des graines d'Orobanche ramosa.

\begin{tabular}{|lc|}
\hline $\begin{array}{l}\text { Année de récolte } \\
\text { des graines d' } \mathbf{0} \text {. ramosa }\end{array}$ & $\begin{array}{c}\text { Fréquence de germination après } \\
\text { récolte } \pm \text { intervalle de confiance à } \mathbf{5} \%\end{array}$ \\
\hline Lot $\mathbf{1 9 9 9}$ & $\mathbf{0 , 8 5} \pm \mathbf{0 , 0 5}$ \\
Témoin 1998 & $0,97 \pm 0,02$ \\
Lot $\mathbf{2 0 0 0}$ & $\mathbf{0 , 8 5} \pm \mathbf{0 , 0 5}$ \\
Témoin 1999 & $0,87 \pm 0,03$ \\
Lot $\mathbf{2 0 0 1}$ & $\mathbf{0 , 8 7} \pm \mathbf{0 , 0 4}$ \\
Témoin 2000 & $0,85 \pm 0,05$ \\
Lot $\mathbf{2 0 0 2}$ & $\mathbf{0 , 9 8} \pm \mathbf{0 , 0 2}$ \\
Témoin 2001 & $0,96 \pm 0,03$ \\
\hline
\end{tabular}

Les fréquences de germination sont calculées sur deux répétitions contenant au minimum 120 graines chacune.

Tableau 2. Évolution sur une année de la fréquence de germination des graines d'Orobanche ramosa récoltées en 2000.

\begin{tabular}{|lc|}
\hline $\begin{array}{l}\text { Nombre de mois } \\
\text { après la récolte des graines }\end{array}$ & $\begin{array}{c}\text { Fréquence de germination } \pm \text { intervalle } \\
\text { de confiance à } \mathbf{5} \%\end{array}$ \\
\hline 0 & $0,85 \pm 0,05$ \\
2 & $0,90 \pm 0,05$ \\
4 & $0,92 \pm 0,05$ \\
6 & $0,91 \pm 0,05$ \\
8 & $0,91 \pm 0,04$ \\
10 & $0,95 \pm 0,03$ \\
12 & $0,85 \pm 0,05$ \\
\hline
\end{tabular}

Les fréquences de germination sont calculées sur deux répétitions contenant au minimum 120 graines chacune.

Tableau 3. Comparaison des fréquences de germination obtenues après récolte et conservation au sec, des graines d'Orobanche ramosa récoltées en 1999, 2000 et 2001.

\begin{tabular}{|lccc|}
\hline $\begin{array}{l}\text { Année } \\
\text { de récolte } \\
\text { des graines } \\
\text { d'0. ramosa }\end{array}$ & $\begin{array}{c}\text { Fréquence } \\
\text { de germination } \\
\text { observée après } \\
\text { récolte } \pm \\
\text { intervalle } \\
\text { de confiance } \\
\text { à } \mathbf{5} \%\end{array}$ & $\begin{array}{c}\text { Fréquence } \\
\text { de germination } \\
\text { observée en } \\
\mathbf{2 0 0 2} \pm \text { intervalle } \\
\text { de confiance } \\
\text { à } \mathbf{5} \%\end{array}$ & $\begin{array}{c}\text { Test } \mathbf{Z} \\
\text { sur évolution } \\
\text { de la fréquence } \\
\text { de germination }\end{array}$ \\
\hline 1999 & $0,85 \pm 0,05$ & $0,93 \pm 0,05$ & \\
2000 & $0,85 \pm 0,05$ & $0,95 \pm 0,04$ & $5,13(\mathrm{~S})$ \\
2001 & $0,87 \pm 0,04$ & $0,96 \pm 0,03$ & $6,76(\mathrm{~S})$ \\
\hline
\end{tabular}

Les fréquences de germination sont calculées sur deux répétitions contenant au minimum 120 graines chacune.

parasite des cultures vivrières) montrent qu'il s'agit de molécules appartenant à la famille des strigolactones [4]. Les semences d'orobanche sont ensuite capables de germer puis de former un lien parasitique avec l'hôte pour des plages de facteurs environnementaux assez larges : de l'anoxie complète à $21 \%$ pour la teneur en oxygène de l'atmosphère, dans une gamme de potentiels hydriques compris entre 0 et - 3,5 $\mathrm{MPa}$ et à des températures comprises entre 1 et $45^{\circ} \mathrm{C}$. L'effet des facteurs environnementaux sur la réussite du préconditionnement puis sur la germination est résumé dans le tableau 4 [6].

Le parasitisme du colza par l'orobanche rameuse avait été surtout observé en sols calcaires. Ce n'est qu'en 2004 que le phénomène a 
Tableau 4. Synthèse des effets des facteurs environnementaux sur le préconditionnement et la germination des graines d'Orobanche ramosa préconditionnées en présence de GR24 (1ppm).

\begin{tabular}{|lll|}
\hline & \multicolumn{1}{c|}{ Préconditionnement } & \multicolumn{1}{c|}{ Germination } \\
\hline Température & $5-30{ }^{\circ} \mathrm{C}$ & $10-25^{\circ} \mathrm{C}$ \\
& $\mathrm{T}^{\circ} \mathrm{C}$ optimale $: 20{ }^{\circ} \mathrm{C}$ & $\mathrm{T}^{\circ} \mathrm{C}$ optimale $: 20{ }^{\circ} \mathrm{C}$ \\
Teneur en $\mathrm{O} 2$ & Dès $1 \% \mathrm{~d}^{\prime} \mathrm{O} 2$ & Dès $3 \% \mathrm{~d}^{\prime} \mathrm{O} 2$ \\
Potentiel Hydrique & Jusqu'à $-2 \mathrm{MPa}$ & Jusqu'à $-3 \mathrm{MPa}$ \\
\hline
\end{tabular}

également été constaté en terres de limons sur trois sites différents [10]. De plus, un test conduit en serre a montré, pour chaque substrat de sol testé (tableau 5), une forte infestation deux mois après la levée du colza. Ces données montrent qu'il n'y a pas de limitation majeure des facteurs environnementaux testés sur le développement de l'orobanche sur colza.

\section{Des hôtes nombreux}

Orobanche ramosa a de nombreux hôtes. De nombreuses espèces sont capables d'induire la germination de l'orobanche puis de constituer une liaison parasitique allant jusqu'à la maturité du parasite et la dissémination des graines. Néanmoins, différents pathotypes d'O.ramosa sont en cours d'étude. On distingue actuellement, sur la base de tests moléculaires, le pathotype T qui s'associerait plutôt au tabac, au chanvre et à la tomate, alors que le pathotype $C$ serait plus spécifique du colza [7]. Ces groupes de pathotypes correspondraient également à des groupes de spécificité d'hôtes sensiblement différents [7]. En associant les différentes sources d'information disponibles, ce sont au moins une cinquantaine $d^{\prime}$ espèces qui peuvent être parasitées par 0 . ramosa [8-10]. Néanmoins, selon les approches et les méthodologies utilisées, l'information n'est pas tout à fait de même nature. II y a, d'une part, les cas où la liaison a été observée à partir d'échantillons au champ, et d'autre part, les cas où la liaison parasitique a été reproduite en conditions contrôlées (serre et chambre de culture) avec observation des stades de développement du parasite. Dans I'approche de Gibot-Leclerc et al., [8], en se basant sur une liste de 82 espèces de plantes adventices présentes dans les champs de colza et après sélection des 22 espèces les plus fréquentes, le parasitisme a été observé au champ sur 11 d'entre elles, parmi lesquelles on retrouve des espèces aussi communes que le géranium disséqué (Geranium dissecum) ou l'alchémille des champs (Aphanes arvensis). On y trouve également deux monocotylédones, le blé (Triticum aestivum) et le ray grass (Lolium multiflorum). Ceci est surprenant dans la mesure où il est généralement admis par les spécialistes que l'orobanche ne s'associe pas aux

Tableau 5. Comparaison des stades de développement d'Orobanche ramosa et de leur nombre, après culture du couple Orobanche ramosa/colza d'hiver cv Zénith, dans des échantillons de terre provenant de Poitou-Charentes.

\begin{tabular}{|c|c|c|c|}
\hline \multirow{2}{*}{$\begin{array}{l}\text { Dates de } \\
\text { prélèvement } \\
\text { des racines } \\
\text { de colza }\end{array}$} & \multicolumn{3}{|c|}{ Type de terre artificiellement infestée } \\
\hline & $\begin{array}{c}\text { Terre } \\
\text { de Bornais } \\
\text { (limon) }\end{array}$ & $\begin{array}{c}\text { Terre } \\
\text { de Champagne } \\
\text { (argilo- } \\
\text { calcaire) }\end{array}$ & $\begin{array}{c}\text { Terre } \\
\text { de marais }\end{array}$ \\
\hline \multirow[t]{4}{*}{$60 \mathrm{JAL}$} & 56 fixations & 158 fixations & $\begin{array}{l}48 \text { tubercules } \\
\text { âgés }\end{array}$ \\
\hline & $\begin{array}{l}20 \text { jeunes } \\
\text { tubercules }\end{array}$ & $\begin{array}{l}54 \text { jeunes } \\
\text { tubercules }\end{array}$ & 25 bourgeons \\
\hline & $\begin{array}{l}38 \text { tubercules } \\
\text { âgés }\end{array}$ & $\begin{array}{l}109 \text { tubercules } \\
\text { âgés }\end{array}$ & $\Sigma: 73$ \\
\hline & $\Sigma: 114$ & $\Sigma: 321$ & \\
\hline
\end{tabular}

Examens réalisés 60 jours après la levée du colza (JAL) sur 20 systèmes racinaires de colza.
Tableau 6. Listes des principales espèces d'adventices hôtes et faux hôtes d'après Boulet et al. 2001 et Gibot-Leclerc et al. 2003 (Observations au champ + tests en conditions contrôlées).

\begin{tabular}{|c|c|c|}
\hline & $\begin{array}{c}\text { D'après Boulet } \\
\text { et al. } 2001\end{array}$ & $\begin{array}{c}\text { D'après Gibot-Leclerc } \\
\text { et al. } 2003\end{array}$ \\
\hline Espèces hôtes & $\begin{array}{l}\text { Galium asparine } \\
\text { Capsella bursa-pastoris } \\
\text { Senecio vulgaris } \\
\text { Sonchus asper } \\
\text { Raphanus raphanistrum }\end{array}$ & $\begin{array}{l}\text { Aphanes arvensis } \\
\text { Crepis foetida } \\
\text { Lolium multiflorum } \\
\text { Mercurialis annua } \\
\text { Calepine irregularis } \\
\text { Euphorbia helioscopia } \\
\text { Geranium dissectum } \\
\text { Picris echinoides } \\
\text { Sonchus oleraceus } \\
\text { Raphanus raphanistrum }\end{array}$ \\
\hline Espèces faux hôtes & $\begin{array}{l}\text { Ridolfia segetum } \\
\text { Veronica persica } \\
\text { Epilobium tetragonum } \\
\text { Geranium dissectum } \\
\text { Lapsana communis } \\
\text { Avena sterilis } \\
\text { Conyza canadense } \\
\text { Ammi majus } \\
\text { Datura stramonium } \\
\text { Cichorium endivia } \\
\text { Anagallis arvensis } \\
\text { Solanum nigrum }\end{array}$ & $\begin{array}{l}\text { Capsella bursa-pastoris } \\
\text { Sinapsis arvensis } \\
\text { Reseda lutea } \\
\text { Anthriscus caucalis }\end{array}$ \\
\hline Espèces immunes & $\begin{array}{l}\text { Amaranthus deflexus } \\
\text { Astragalus boeticus } \\
\text { Chenopodium album } \\
\text { Convolvulus arvensis } \\
\text { Echinochloa crus-galli } \\
\text { Fumaria officinalis } \\
\text { Mercurialis annua ssp } \\
\text { annua } \\
\text { Plantago major ssp major } \\
\text { Polygonum persicaria } \\
\text { Polygonum aviculare ssp } \\
\text { aviculare } \\
\text { Portulaca oleracea } \\
\text { Stellaria media ssp media }\end{array}$ & Polygonum aviculare \\
\hline
\end{tabular}

monocotylédones, mais seulement aux dicotylédones. Pour 9 de ces 11 espèces, un test en serre a permis de reproduire le parasitisme. Pour Lolium multiflorum le lien parasitique a pu être reproduit en serre, même si le nombre de hampes florales par plante hôte est resté limité à quelques individus [8]. Il a également été possible de reproduire le parasitisme avec des plantes comme la capselle bourse à pasteur (Capsella bursa pastoris), la renouée persicaire (Polygonum persicaria) et la ravenelle (Raphanus raphanistrum), mais en obtenant des hampes florales que pour la dernière espèce citée.

Les résultats des tests effectués en serre à l'université de Nantes portent sur 35 espèces adventices [9]. Treize d'entre elles sont considérées comme immunes, et donc incapables de contribuer au développement de l'orobanche. Dans le cas du cerfeuil (Anthriscus causalis), une germination tardive de l'orobanche est observée, mais sans fixation. Pour 7 espèces, Boulet et al. [9], observent une fixation accompagnée d'une nécrose, et donc un arrêt du parasitisme. Pour 5 espèces, il y a une fixation, mais jamais d'émergence. Elles peuvent alors être considérées comme des faux-hôtes (tableau 6). Cependant, des variations de conditions de milieu 
Tableau 7. Résultats des essais variétés conduits en Poitou-Charentes en 2000-2001 (non récolté- 270 à 360 plantes $/ \mathrm{m}^{2}$ ) et en $2001-2002$ (130 à 200 plantes $/ \mathrm{m}^{2}$ ).

\begin{tabular}{|c|c|c|c|c|c|}
\hline Variétés & $\begin{array}{c}\text { 2000-2002\% réduction } \\
\text { peuplement d'orobanches } \\
\text { p/ témoin adjacent (cv Zénith) }\end{array}$ & $\begin{array}{c}\text { Rendement Qx/Ha } \\
\text { (Newman et Keuls 5\%) }\end{array}$ & $\begin{array}{c}\text { Gravité } \\
\text { au } 16 \text { mai } 2002\end{array}$ & $\begin{array}{c}2000-2001 \\
\text { Gravité au } 20 \text { mars } 2001\end{array}$ & $\begin{array}{c}\text { Gravité } \\
\text { au } 16 \text { mai } 2001\end{array}$ \\
\hline Zénith & $0 \%$ & $13,2 \mathrm{a}$ & 5 & 3 & 7 \\
\hline Capitol & $0 \%$ & $11,6 \mathrm{a}$ & 6 & 2 & 9 \\
\hline Extra & $40 \%$ & $25,9 \mathrm{~b}$ & 2 & 1 & 4 \\
\hline Parent Mâle & $75 \%$ & $24,9 b$ & 1 & 1 & 1 \\
\hline $\begin{array}{l}\text { Parent } \\
\text { Femelle }\end{array}$ & $0 \%$ & $11,7 a$ & 7 & 3 & 9 \\
\hline Explus & $0 \%$ & $23,8 \mathrm{~b}$ & 2 & & \\
\hline Lutin & $82 \%$ & $21,7 \mathrm{~b}$ & 1 & & \\
\hline Talent & $87 \%$ & $31,8 \mathrm{~b}$ & 1 & & \\
\hline Constant & $82 \%$ & $30,3 b$ & 1 & & \\
\hline Cordial & $85 \%$ & $28,9 \mathrm{~b}$ & 1 & & \\
\hline Standy & $25 \%$ & $24,0 \mathrm{~b}$ & 2 & & \\
\hline
\end{tabular}

Echelle de gravité : 1 sain/9 très parasité.

pourraient induire l'émergence. Ceci peut expliquer certaines divergences de résultats entre équipes. Enfin, Boulet et al. [9] identifient plusieurs espèces considérées comme de vrais hôtes (Capsella bursa pastoris, Galium aparine, Senecio vulgaris, Sonchus asper, et dans une moindre mesure Raphanus raphanistrum).

Plusieurs convergences de résultats sont notables : les deux équipes sont d'accord pour constater l'absence de parasitisme avec émergence pour la renouée des oiseaux (Polygonum aviculare), le réséda jaune (Reseda lutea) et l'ammi majus (Ammi majus). Elles constatent également toutes les deux que le laiteron rude et le laiteron maraîcher (genre Sonchus) sont de vrais hôtes, ainsi que la ravenelle (Raphanus raphanistrum). II y a un désaccord pour trois espèces importantes : le géranium disséqué (Geranium dissectum), la mercuriale annuelle (Mercurialis annua), et le liseron des champs (convolvulus arvensis). Pour ces trois espèces, il pourrait donc $y$ avoir interaction avec les conditions des tests, et ceux-ci devront être refaits dans différentes conditions.

De nouvelles observations faites récemment en 2004 au champ [10] ont montré également que le melon (Cucumis melo, variété inconnue) peut être un hôte. II faudra, dans ce cas, faire la vérification par un test de parasitisme en conditions contrôlées. Robin [10] a également pu confirmer la présence du parasite sur plusieurs espèces d'adventices qui peuvent être hôtes: laiteron maraîcher (Sonchus oleraceus), ravenelle (Raphanus raphanistrum), helminthie fausse vipérine (Picris echioides), géranium disséqué (Geranium dissectum), gaillet gratteron (Galium aparine), calépine (Calepine irregularis), capselle bourse à pasteur (Capsella bursa pastoris).

\section{Climat plus ou moins favorable}

L'orobanche rameuse est un parasite potentiel de nombreuses espèces dont les durées de cycle de développement diffèrent. Ainsi, associée à Arabidopsis thaliana, l'orobanche est capable de faire un cycle complet en quelques semaines. Par contre, le cycle peut durer quelques mois sur la tomate ou le tabac, et 10 ou 11 mois sur colza. Par ailleurs, si le cycle du parasite s'adapte à la durée du cycle de développement de l'hôte, il existe des variations selon la température. Ainsi, en cas d'hiver et de printemps doux, l'émergence de l'orobanche se fera plus tôt au cours du cycle et pourra de ce fait se développer abondamment et produire davantage de semences. Lorsqu'une parcelle est abondamment parasitée, et lorsque l'émergence a lieu précocement (dès mars), la nuisibilité exercée sur le rendement du colza est très importante (2000 et 2001). Elle peut, dans certains cas, aller jusqu'à l'absence de récolte, avec une dizaine de hampes florales d'orobanche par plante de colza. Elle l'est moins (de l'ordre de $10 \%$ ) lorsque l'émergence est plus tardive (fin mai ; 1999 ou 2004 par exemple). Le positionnement relatif des stades de développement du parasite et de son hôte sur une échelle thermique permet, d'une part, de confirmer une stabilité des stades du parasite par rapport à ceux d'un hôte donné et, d'autre part, de définir avec un indicateur exprimé en somme de températures le stade de développement du parasite alors que celui-ci est encore souterrain et invisible pour l'agriculteur.

\section{Les ébauches de solutions}

Les préconisations à court terme visent soit à avantager la plante hôte par rapport au parasite dans leur compétition nutritionnelle, soit à éviter au maximum l'entretien ou l'augmentation du stock semencier du parasite dans le sol. En favorisant le développement de l'hôte, et donc en augmentant ses capacités nutritionnelles (carbone, azote, etc.), on peut penser que la part relative prélevée par le parasite sera plus faible, et qu'en conséquence I'hôte sera moins pénalisé. Ceci a amené à recommander l'utilisation de matériel hybride plus vigoureux, à diminuer les densités de semis de l'hôte et à éviter les semis trop précoces pour rechercher des conditions climatiques freinant davantage le développement du parasite. Des essais de comportement variétal en situation infestée ont permis de tester l'hypothèse variétale avec succès, à condition toutefois que la densité d'orobanche ne soit pas trop forte. Le tableau 7 illustre les résultats obtenus deux années de suite dans des parcelles infestées de Poitou-Charentes. La forte infestation de la première année (270 à 360 orobanches par mètre carré) n'a pas permis la récolte de l'essai, alors que cela a été possible l'année suivante. Pour les deux essais, on remarque effectivement un avantage pour le matériel hybride (composites hybrides lignées et hybrides restaurés) sur lequel les notes de gravité sont plus faibles, les pourcentages de réduction de population d'orobanche par rapport au témoin adjacent plus forts. Pour l'essai récolté, on constate qu'il y a une nette distinction de niveaux de productivité entre matériels hybrides et lignées. Par ailleurs, dans ces essais figuraient également les lignées parentales de l'un des hybrides restaurés. La lignée mâle a un comportement vis-à-vis de l'orobanche qui s'apparente à celui des autres matériels hybrides. La spécificité de cette lignée restauratrice de fertilité mâle Ogu-Inra est d'avoir intégré un fragment de génome de radis qui apporte ce caractère de restauration. Cette restauration est souvent accompagnée d'effets indésirables tels que la baisse de fertilité femelle chez l'hybride, ou des modifications du niveau de glucosinolates dans les graines. On ne peut pas non plus exclure I'hypothèse que cette introgression «radis » ait également 


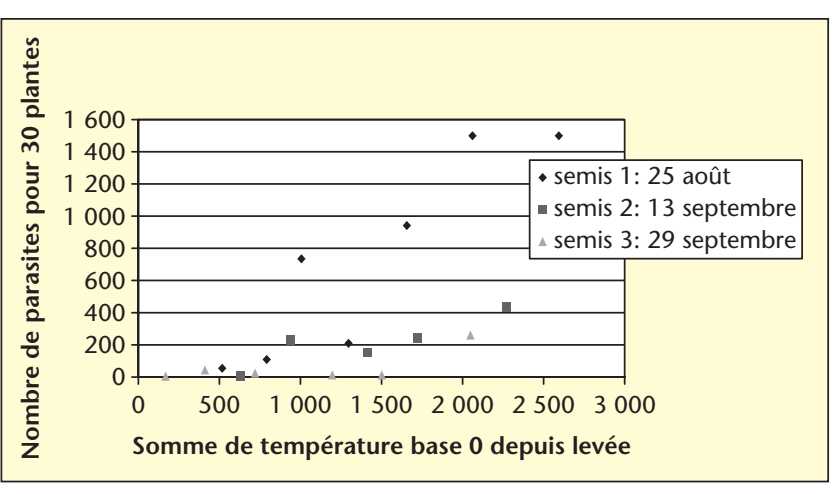

Figure 2. Cinétique d'installation de l'orobanche en fonction de la date de semis. Essai au champ 2000-2001.

apporté un élément favorable pour la tolérance à l'orobanche. Néanmoins, la sélection poursuivant ses travaux pour réduire au maximum les effets indésirables, en réduisant l'introgression le plus possible aux séquences responsables de la restauration, il n'est pas certain que l'avantage introduit pour le contrôle de l'orobanche subsiste dans les générations plus récentes d'hybrides restaurés Ogu-Inra.

L'effet d'un décalage des dates de semis a été testé en 1999-2000 et en 2000-2001. La première année un décalage de la date de semis de 3 semaines (24 septembre au lieu du 30 août) a permis de réduire de 279 à 38 le nombre d'orobanches au mètre carré. Par contre, le rendement a été fortement affecté dans les deux cas avec peu d'écart (respectivement 17,9 et 18,6 qx/ha). La deuxième année, trois dates de semis ont été testées. Les résultats sont présentés dans la figure 2 et montrent que la cinétique de mise en place du parasitisme a été très fortement freinée. Par contre, un retard trop important de la date de semis expose l'agriculteur à d'autres risques tels que l'enherbement et la contamination par le Phoma. Dans le cas présent, les dates de semis du 13 et du 29 septembre ont produit de petits colzas peu compétitifs vis-à-vis des plantes adventices.

Des essais de traitement herbicides ont été menés par le Cétiom entre 1998 et 2002 [11]. Après une première année qui a permis de trier une quinzaine de molécules herbicides systémiques sur une base de sélectivité pour le colza, trois molécules ont ensuite fait l'objet d'expérimentations plus complètes pour déterminer dose et période d'application à la fois sur l'efficacité et la sélectivité pour le colza. Deux molécules présentent un intérêt, le glyphosate (2 fois 0,3 litre/ha en entrée puis pendant I'hiver) et l'hydrazine maléique (régulateur de croissance utilisé pour supprimer les germes pendant la conservation des légumes, ou comme ébourgeonnement chimique sur tabac) (2 fois $750 \mathrm{~g} / \mathrm{ha}$ aux mêmes périodes). Cependant, si les efficacités atteintes sont satisfaisantes, on est la plupart du temps à la limite de la sélectivité. Compte tenu de l'équilibre très instable entre sélectivité et efficacité, ce type de solution ne pourra pas satisfaire aux critères et aux conditions d'obtention d'une autorisation de mise sur le marché, et ne pourra donc en aucun cas être conseillé aux agriculteurs. Néanmoins, une nouvelle formulation de l'hydrazine maléique permettrait une amélioration de la sélectivité (Source Anitta, Institut technique du Tabac).

D'autres mesures à court terme sont envisageables. Compte tenu du nombre important d'hôtes pouvant contribuer à la multiplication de I'orobanche, des recommandations en matière de qualité de désherbage avant floraison sont nécessaires. Les programmes de désherbage s'efforceront de cibler en priorité les espèces répertoriées comme hôtes potentiels. La vigilance en ce domaine devra également s'exercer vis-à-vis des bordures de parcelles, des intercultures et des jachères, autant de milieux dans lesquels des adventices hôtes sont susceptibles de contribuer à la multiplication de l'orobanche. Le parasite apparaissant souvent en foyer dans une parcelle, il ne faudra pas non plus hésiter à détruire avec un désherbant total un foyer important. II est en effet préférable de perdre quelques centaines de $\mathrm{m}^{2}$ de récolte, plutôt que de contribuer à la dissémination de semences d'orobanche. Une diversification des espèces présentes dans la rotation, avec un retour moins fréquent d'espèces pouvant être des hôtes au profit d'espèces étant plus probablement des faux hôtes (plantes capables de provoquer la germination, mais sans association parasitique allant à terme) doit également pouvoir constituer une mesure utile au contrôle de l'extension du phénomène.

À plus long terme, on envisage d'exploiter plus rationnellement l'utilisation dans la rotation et dans les intercultures, des espèces faux hôtes qui peuvent contribuer à faire baisser significativement le stock semencier $d^{\prime}$ orobanche du sol. Pour cela, il est nécessaire de poursuivre les efforts $d^{\prime}$ 'inventaire et de caractérisation des espèces hôtes pouvant être utilisées comme plantes pièges dans l'interculture, et les espèces faux hôtes utilisables dans la rotation en culture principale ou en interculture. La conception de rotations permettant de diminuer significativement le stock semencier du parasite dans le sol devrait pouvoir s'appuyer sur un travail de modélisation du stock semencier permettant d'explorer le champ des possibles.

Il est également envisageable de sélectionner des colzas moins sensibles. Ce recours à la génétique a été largement utilisé sur tournesol pour la résistance à Orobanche cumana, mais principalement avec des introductions successives de gènes de résistances spécifiques qui ont ensuite été régulièrement contournés [12]. Nos essais au champ ainsi que des études préliminaires réalisées à l'université de Nantes [13] suggèrent qu'il existe une variabilité génétique chez le colza pour la tolérance à l'orobanche. Ceci doit permettre une étude génétique de recherche de facteurs de résistance.

Une autre démarche possible est l'utilisation de plantes hôtes résistantes à un herbicide à large spectre appliqué en post-levée et suffisamment systémique et efficace contre l'orobanche. Des colzas OGM résistants au glyphosate et des colzas résistants (obtenus par mutagénèse) aux imidazolinones ont été testés avec succès [14]. Aujourd'hui des variétés de tournesol résistantes aux imidazolinones sont commercialisées en Turquie, et bientôt en Espagne, et permettent une lutte efficace contre l'orobanche. Néanmoins, reste posée la question de la durabilité de cette solution, dans la mesure où la famille des imidazolinones est la famille $\mathrm{d}$ 'herbicide pour laquelle la liste des espèces sauvages présentant des résistances naturelles est la plus longue [15].

Remerciements. Les auteurs remercient leurs collègues du CETIOM qui ont participé aux travaux sur l'orobanche, et particulièrement ceux de la station d'expérimentation de Surgères [17] qui ont réalisé la plupart des expérimentations au champ et des prélèvements en parcelles agriculteurs. La thèse de S. Gibot-Leclerc a été financée par le CETIOM et par le conseil régional de Poitou-Charentes.

\section{RÉFÉRENCES}

1. PERNY A. La phélipée rameuse plante parasite du colza. Phytoma Défense Végétaux 1991 ; 424 : 45-6.

2. IZARD C, HITIER H. Obtention de la germination in vitro des graines d'Orobanche du Tabac. Ann Inst Expérimental Tabac Bergerac 1953 ; 1 : 47-56.

3. CHAUVEL B, DESSAINT F, LONCHAMP JP, GASQUEZ J. Cinq élues et des candidates, enquête sur les mauvaises herbes envahissantes en grandes cultures en France. Phytoma Défense Végétaux 2005 ; 578 : 16-20.

4. GIBOT-LECLERC $S$. Etude épidémiologique, écophysiologique et agronomique du couple Orobanche ramosa L. / Brassica napus L. Thèse de Doctorat Université Paris VI Pierre et Marie Curie, 2004.

5. SAGHIR AR. Dormancy and germination of Orobanche seeds in relation to control methods. In : Biology and control of Orobanche, Proceedings of a workshop Wagueningen. 1986, S) ter Borg (ed), Pays Bas, p. 25-34. 
6. GIBOT-LECLERC S, CORBINEAU F, SALLE G, CÔME D. Responsiveness of Orobanche ramosa L. seeds to GR24 as related to temperature, Oxygen avaibility and water potential during preconditioning and subsequent germination. Plant Growth Regul $2004 ; 43: 63-71$.

7. BeNHARRAT H, VERONESI C, THEODET C, THALOUARN P. Orobanche species and population discrimination using intersimple sequence repeat (ISSR). Weed Res $2002 ; 42: 470-5$.

8. GIBOT-LECLERC S, BRAULT M, PINOCHET X, SALLE G. Rôle potentiel des plantes adventices du colza d'hiver dans l'extension de l'orobanche rameuse en Poitou-Charentes. Comptes Rendus Biologies Paris 2003 ; $326: 645-58$.

9. BOULET C, LABROUSSE P, ARNAUD MC, ZEHHAR N, FER A. Weed species present various responses to Orobanche ramosa. In : A. Fer, P. Thalouarn, DM Joel, LJ Musselman, C Parker, JAC Verkleij (eds). Proceedings of the seventh international parasitic Weed Symposium. Faculté des Sciences de Nantes: 228-231, 2001.

10. ROBIN P. Evaluation et compréhension des facteurs d'extension de l'orobanche rameuse en region Poitou-Charentes. Mémoire de DESS productions végétales ENSAR-Université Rennes $1 ; 2004$.
11. CHICOTE-EMEIDI H, LEVENGEUX T, ARJAURE G, PINOCHET $X$, PILORGE E. Search for chemicals able to control the parasitic plant Orobanche ramosa $\mathrm{L}$. and enough selective of a field winter oilseed rape. $11^{\text {th }}$ International rapeseed Congress. Copenhagen Danmark $2003 ; 4: 1201-3$.

12. MELERO-VARA JM, DOMINGUEZ J, FERNANDEZ-MARTINEZ JM. Update on sunflower broomrape situation in spain racial status and sunflower breeding for resistance. Helia $2000 ; 23: 15-56$.

13. ZEHHAR N, LABROUSSE P, ARNAUD MC, BOULET C, BOUYA D, FER A. Study of resistance to Orobanche ramosa in host (Oilseed rape and carrot) and non-host (maize) plants. Eur J Plant Pathol 2003 ; 109 : 75-82.

14. JOEL DM, PORTNOY VH, GRESSEL J, AMSELLEM Z. Treatment of transgenic Herbicide-resistant seeds for broomrape Control. Abstract of papers presented at the $15^{\text {th }}$ conference of the weed science society of Israel Bet Dagan March 1998. Phyoparasitica $27: 7$.

15. WWW. weedscience. org/in. asp. 2005. 